\title{
Quantitative analysis of intermolecular interactions in 2,2'-((4-bromophenyl)methylene)bis(3-hydroxy-5,5- dimethylcyclohex-2-en-1-one): insights from crystal structure, PIXEL, Hirshfeld surfaces and QTAIM analysis
}

\author{
SUBBIAH THAMOTHARAN ${ }^{\mathrm{a}, *}$, JAGATHEESWARAN KOTHANDAPANI ${ }^{\mathrm{b}}$, \\ SUBRAMANIAPILLAI SELVA GANESAN ${ }^{\mathrm{b}}$, NATARAJAN S VENKATARAMANAN ${ }^{\mathrm{b}}$, \\ SHANKAR MADAN KUMAR ${ }^{c}$, KULLAIAH BYRAPPA ${ }^{\mathrm{d}}$, JUDITH PERCINO ${ }^{\mathrm{e}}$ and \\ FERNANDO ROBLES ${ }^{\mathrm{e}}$ \\ ${ }^{a}$ Biomolecular Crystallography Laboratory, Department of Bioinformatics, School of Chemical and \\ Biotechnology, SASTRA University, Thanjavur, Tamilnadu 613 401, India \\ ${ }^{\mathrm{b}}$ Department of Chemistry, School of Chemical and Biotechnology, SASTRA University, Thanjavur, Tamilnadu \\ 613 401, India \\ ${ }^{c}$ PURSE Laboratory, Mangalagangotri Mangalore University, Mangalore, Karnataka 574 199, India \\ ${ }^{\mathrm{d}}$ Department of Materials Science, Mangalore University, Mangalore, Karnataka 574 199, India \\ ${ }^{\text {e}}$ Lab. de Polímeros, Centro de Química, Instituto de Ciencias, Benemérita Universidad Autónoma de Puebla, \\ Complejo de Ciencias, ICUAP, Edif. 103H, 22 Sur y San Claudio, C.P. 72570 Puebla, Puebla, Mexico \\ E-mail: thamu@scbt.sastra.edu
}

MS received 16 October 2017; revised 28 December 2017; accepted 29 December 2017; published online 7 February 2018

\begin{abstract}
.
The crystallographic study of 2,2'-((4-bromophenyl)methylene)bis(3-hydroxy-5,5-dimethylcyclohex-2-en-1one) reveals that the compound crystallizes in the centrosymmetric space group $P 2_{1} / c$. In the solid state, the structure of the title compound exhibits two strong intramolecular $\mathrm{O}-\mathrm{H} \cdots \mathrm{O}$ hydrogen bonding interactions. Further, molecules of the title compound are self-assembled by weak intermolecular $\mathrm{C}-\mathrm{H} \cdots \mathrm{O}, \pi \cdots \pi$ and $\mathrm{H} \cdots \mathrm{H}$ and $\mathrm{C}-\mathrm{H} \cdots \mathrm{Br}$ contacts. Various intermolecular interaction that exist in the crystal structure and their energetics are quantified using PIXEL, DFT and QTAIM analyses. Six different motifs are identified from the PIXEL calculation. Lattice energy calculation suggests that the dispersion energy has the highest contribution for the crystal formation. The relative contributions of various intermolecular contacts in the title compound and its closely related analogs are evaluated using Hirshfeld surface analysis and the decomposed fingerprint plots. The common packing features exist between the title compound and its related analogs are identified. The quantitative molecular electrostatic potential surface diagram depicts the potential binding sites which are in good agreement with the crystal structure of the title compound. The structures of title compound in gas and solvent phases are compared with the experimental structure and reveals that they are superimposed very well. The vibrational modes of the monomer and four most stabilized dimers are characterized using both the experimental and DFT calculations. The UV-Vis spectrum is calculated using time dependent-DFT (TD-DFT) method and compared with experimental spectrum. The results indicate that the calculated energy of absorbance and oscillator strength correlate well with the experimental data.
\end{abstract}

Keywords. Cyclohexene; hirshfeld surface; keto-enol hydrogen bonding; dispersion interactions; PIXEL; QTAIM; TD-DFT.

\footnotetext{
*For correspondence

Electronic supplementary material: The online version of this article (https://doi.org/10.1007/s12039-018-1421-8) contains supplementary material, which is available to authorized users.
} 


\section{Introduction}

Derivatives of 2,2'-arylmethylene bis(3-hydroxy-5,5dimethyl-2-cyclohexene-1-ones) were used as precursors for the synthesis of heterocyclic compounds, especially xanthenes and acridinediones, and some of these derivatives were used as laser dyes. ${ }^{1,2}$ They also showed interesting biological activities such as antioxidant, ${ }^{3}$ tyrosinase inhibition, ${ }^{4}$ anti-viral ${ }^{5}$ and anti-bacterial activities. ${ }^{6}$ In view of this, these compounds were synthesized from aromatic aldehydes with dimedone (5,5dimethyl-1,3-cyclohexanedione) under different conditions, using different catalysts. ${ }^{7-12}$ However, the title compound and its closely related analogs were prepared by an inexpensive zinc chloride as catalyst in environmentally benign water medium. ${ }^{9}$ Moreover, the product was isolated from the reaction medium by simple filtration. Thus, a green protocol was used for the synthesis of the title compound. It is of interest to note that several arylmethylene bis(3-hydroxy-5,5-dimethylcyclohex-2en-1-one) derivatives have been crystallographically analyzed. ${ }^{13-20}$ It is known that the crystal packing is predominantly controlled by strong intermolecular hydrogen bonds such as $\mathrm{O} / \mathrm{N}-\mathrm{H} \cdots \mathrm{O} / \mathrm{N}$ due to the presence of strong donor and acceptor atoms. ${ }^{21,22}$ Moreover, the weak intermolecular interactions such as $\mathrm{C}-\mathrm{H} \cdots \mathrm{O} / \mathrm{N},{ }^{23,24} \mathrm{C}-\mathrm{H} \cdots$ halogens, ${ }^{25,26} \mathrm{C}-\mathrm{H}$ $\cdots \pi^{27}$ and $\pi \cdots \pi^{28}$ interactions can also interplay a crucial role in controlling the crystal packing in the absence of strong donors and acceptors. In the case of title compound and its closely related analogs, the available donor $(\mathrm{O}-\mathrm{H})$ and acceptor $(\mathrm{C}=\mathrm{O})$ are fully participated in two intramolecular $\mathrm{O}-\mathrm{H} \ldots \mathrm{O}$ hydrogen bonding interactions. In this situation, a study to understand how other weak interactions help to stabilize the crystal packing of the title compound and its analogs gains importance.

In this work, we report the crystal and molecular structure of 4-bromo derivative, I, namely 2,2'-((4bromophenyl)methylene)bis(3-hydroxy-5,5-dimethylcyclohex-2-en-1-one) to understand the nature of intermolecular interactions present in the molecule. We also present a detailed quantitative analysis of energetics of various molecular pairs identified from the crystal structure of compound I using the PIXEL method. Further, molecular conformation and lattice energies of the compound I are compared with the structures of unsubstituted and several para- substituted derivatives to understand the influence of substituents on the molecular conformation and crystal packing features.

Moreover, the relative contributions of various intermolecular interactions present in the compound $\mathbf{I}$ and its analogs were quantified using the Hirshfeld surface
(HS) and fingerprint plots (FP). The structure of the compound I was optimized in the gas phase as well as in the ethanol solvent using the dispersion corrected M05-2X functional with 6-31+G(d) basis set. The topological properties of various interactions observed in the crystal structure of the compound I was carried out using the quantum theory of atoms-in-molecule (QTAIM) approach. ${ }^{29,30}$ Furthermore, to elucidate the optical properties of the compound $\mathbf{I}$, we have measured UV-Vis spectrum and the experimental spectrum is compared with the simulated spectrum computed using the time-dependent DFT (TD-DFT) calculation.

\section{Experimental}

\subsection{Synthesis and crystallization}

To a stirred homogeneous solution of dimedone (140 mg, 1 $\mathrm{mmol})$ in water $(3 \mathrm{~mL})$, 4-bromobenzaldehyde $(93 \mathrm{mg}, 0.5$ $\mathrm{mmol}$ ) and $\mathrm{ZnCl}_{2}(17 \mathrm{mg}, 0.125 \mathrm{mmol})$ were successively added and stirred at room temperature. The reaction was monitored by TLC. After completion of the reaction, the solid product was isolated by simple filtration, washed with water $(2 \times 10 \mathrm{~mL})$ and dried. The crude product was washed with hexane $(2 \times 5 \mathrm{~mL})$ and dried under vacuum. Single crystals of title compound $\mathbf{I}$ was produced from ethanol by the slow evaporation method.

\subsection{Spectral characterization}

The solid-state FT-IR spectrum of the compound I was measured in the frequency region of $4000-400 \mathrm{~cm}^{-1}$ on a Perkin Elmer FT-IR spectrophotometer using the $\mathrm{KBr}$ pellet technique. The solid-state Raman spectrum was recorded using the iRaman plus Raman spectrometer (B \& W Tek, USA) and the $532 \mathrm{~nm}$ laser line was used for excitation. The UV-Vis absorption spectrum was measured on an Eppendorf Biospectrophotometer in ethanol solvent.

\subsection{Single crystal $X$-ray diffraction}

The X-ray intensity data was collected for the compound I on a Rigaku AFC12 Saturn724+ diffractometer. The crystal structure of the compound I was solved by the SIR92 program ${ }^{31}$ and all the non-hydrogen atoms were refined anisotropically using the SHELXL2014 program. $^{32}$ The hydrogen atoms were placed in idealized geometrical positions $(\mathrm{C}-\mathrm{H}=$ $0.93-0.97 \AA$ and $\mathrm{O}-\mathrm{H}=0.82 \AA$ ) and constrained to ride on their parent atoms. The methyl hydrogen atoms were allowed to rotate freely about the $\mathrm{C}-\mathrm{C}$ bonds. The ORTEP and packing figures were produced using the program MERCURY. ${ }^{33}$ The crystal data and refinement parameters for the compound $\mathbf{I}$ are summarized in Table 1. 
Table 1. Crystal data and refinement parameters for the compound $\mathbf{I}$.

\begin{tabular}{ll}
\hline Formula & $\mathrm{C}_{23} \mathrm{H}_{27} \mathrm{BrO}_{4}$ \\
Formula weight & 447.35 \\
Size $\left(\mathrm{mm}^{3}\right)$ & $0.25 \times 0.25 \times 0.20$ \\
Crystal system & Monoclinic \\
Space group, $Z$ & $P 2{ }_{1} / \mathrm{c}, 4$ \\
a $(\AA)$ & $16.394(3)$ \\
b $(\AA)$ & $10.8984(18)$ \\
c $(\AA)$ & $12.0467(19)$ \\
$\alpha\left(^{\circ}\right)$ & 90 \\
$\beta\left(^{\circ}\right)$ & $99.139(7)$ \\
$\gamma\left({ }^{\circ}\right)$ & 90 \\
Temperature $(\mathrm{K})$ & $296(2)$ \\
Volume & $2125.0(6)$ \\
Wavelength $(\AA)$ & 0.71075 \\
Density $\left(\mathrm{Mgm}{ }^{-3}\right)$ & 1.398 \\
No. of reflections collected & 14172 \\
No. of unique reflections & 3715 \\
$2 \theta$ max $\left({ }^{\circ}\right)$ & 50 \\
No. of parameters & 260 \\
R1, wR2 $(\mathrm{I}>2 \sigma(\mathrm{I}))$ & $0.0571,0.117$ \\
Goodness of fit & 1.025 \\
\hline
\end{tabular}

\subsection{Hirshfeld surface analysis, PIXEL energy calculation and crystal packing features}

To investigate the similarities and differences in the crystal packing amongst closely related analogs, the Hirshfeld surface (HS) and the decomposed 2D fingerprint plots were produced for the compound I using the program CrystalExplorer 3.1. ${ }^{34}$ A comparative analysis of Hirshfeld surfaces mapped with different properties such as $d_{\mathrm{i}}, d_{\mathrm{e}}, d_{\text {norm }}$, shape index and curvedness of the compound $\mathbf{I}$ and its 4-substituted analogs was performed. To quantify the energies associated with various intermolecular interactions present in the crystal structure of I, PIXEL calculation ${ }^{35-37}$ was carried out as reported earlier. ${ }^{38-41}$ Briefly, the distances involving hydrogen atoms are moved to their neutron values $(\mathrm{C}-\mathrm{H}=1.089 \AA$ and $\mathrm{O}-\mathrm{H}=0.993 \AA$ ) and the resultant geometry used for the calculation. The electron density of the molecule has been obtained at the MP2/6-31G** level of theory using Gaussian $09 .{ }^{42}$ The total energy calculated by the PIXEL method is partitioned into the corresponding Coulombic, polarization, dispersion and repulsion energies. Six motifs (I to VI) were identified from the crystal structure based on their interaction energy calculated by PIXEL method. To identify the common packing features exist between the compound $\mathbf{I}$ and its closely related analogs, XPAC2.0 program was used. ${ }^{43-45}$ This program provides the dissimilarity index ' $\mathrm{X}$ ' which is a measure of how far the two crystal structures deviate from perfect geometrical similarity.

\subsection{Computational details}

The initial geometry for optimization was obtained from the crystal structure. The monomer I was optimized in gas and solvent phases using the Gaussian 09 program package. ${ }^{42}$ The solvent (ethanol) effect was introduced in the calculation using the conductor-like polarizable continuum model (CPCM) method. The optimized structures were found to have no imaginary frequencies, implying that the structures are global minima in their potential energy surface. For optimization, M05-2X functional ${ }^{46}$ was used along with $6-31+G(d)$ basis set. The choice of M05-2X functional was selected based on the previous reports which were able to predict correct binding energies between molecules with weak interactions. ${ }^{40,41,47}$ The interaction energy $E_{\text {int }}$ was computed for all six motifs at their crystal structure geometry using the supramolecular approach. The $E_{\text {int }}$ was corrected for basis set superposition error (BSSE) using the counterpoise method. ${ }^{48}$ The time dependent-DFT (TD-DFT) calculation in the ethanol solvent was carried out using the time-dependent Kohn-Sham formalism. ${ }^{49}$ The topological parameters were calculated using the AIMALL package ${ }^{50}$ on selected molecular pairs. For this purpose, the DFT calculation (with density $=$ current keyword) for these molecular pairs at their crystal geometry (with bond lengths involving $\mathrm{H}$ atoms adjusted to typical neutron diffraction values), was performed at the M05-2X/6-31+G(d) level of theory.

\section{Results and Discussion}

\subsection{Molecular geometries}

The compound I crystallizes in the centrosymmetric monoclinic space group $P 2_{1} / \mathrm{c}$ with one molecule in the asymmetric unit. The ORTEP diagram of the compound $\mathbf{I}$ with the atom-numbering scheme is shown in Figure 1(a). The molecule comprises three ring systems of which two of them are cyclohexene rings ( $\mathrm{B}$ and C). Each cyclohexene ring has one keto and an enol group. These keto and enol groups form two strong intramolecular $\mathrm{O}-\mathrm{H} \ldots \mathrm{O}$ hydrogen bonding interactions. In the compound $\mathbf{I}$, the $\mathrm{H} \cdots \mathrm{O}$ distances are $1.596(\mathrm{H} 3 \mathrm{O} \ldots \mathrm{O} 2)$ and $1.691 \AA(\mathrm{H} 1 \mathrm{O} \ldots \mathrm{O} 4)$ and the hydrogen bonding angles are $159(\angle \mathrm{O} 1-\mathrm{H} 1 \mathrm{O} \cdots \mathrm{O} 4)$ and $164^{\circ}(\angle \mathrm{O} 3-\mathrm{H} 3 \mathrm{O} \cdots \mathrm{O} 2)$. Rings $\mathrm{B}$ and $\mathrm{C}$ have an envelope conformation as evidenced by the Cremer and Pople ${ }^{51}$ puckering parameters [ring B: $Q=$ $0.460(5) \AA, q_{2}=0.422(5) \AA, q_{3}=-0.184(5) \AA, \theta=$ $113.6(6)^{\circ}$ and $\varphi_{2}=356.0(7)^{\circ}$ for the atom sequence $\mathrm{C} 2-\mathrm{C} 3-\mathrm{C} 4-\mathrm{C} 5-\mathrm{C} 8-\mathrm{C} 9$ and ring C: $Q=0.457(5)$ $\AA, q_{2}=0.412(4) \AA, q_{3}=-0.199(5) \AA, \theta=$ $115.8(6)^{\circ}$ and $\varphi_{2}=358.8(6)^{\circ}$ for the atom sequence $\mathrm{C} 10-\mathrm{C} 11-\mathrm{C} 12-\mathrm{C} 13-\mathrm{C} 16-\mathrm{C} 17]$. The dihedral angle between ring $\mathrm{A}$ and the rings $\mathrm{B} / \mathrm{C}$ are 65.2(1) and 

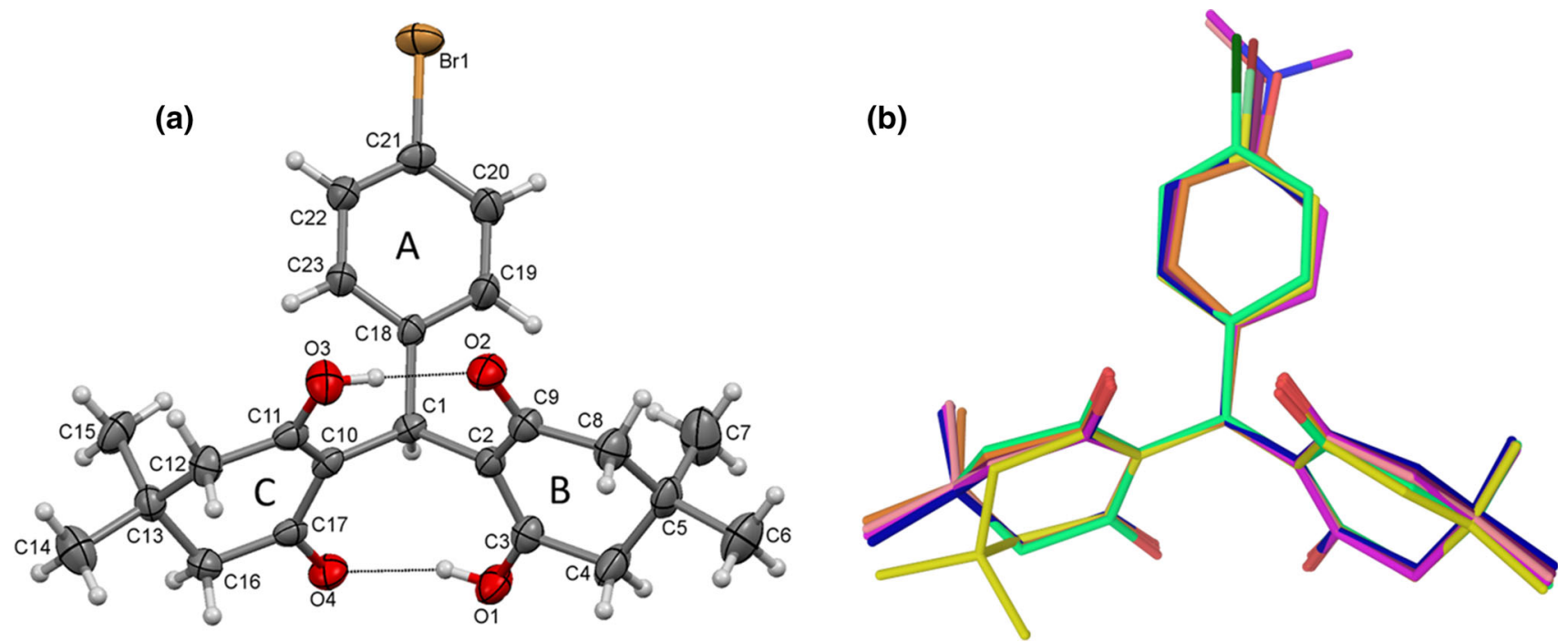

Figure 1. ORTEP diagram, atom-labelling scheme and ring labelling for title compound I. Thermal ellipsoids are drawn at $50 \%$ probability level. Intramolecular $\mathrm{O}-\mathrm{H} \cdots \mathrm{O}=\mathrm{C}$ hydrogen bonds which lock the molecular conformation are depicted (b) Structural superimposition of $\mathbf{I}$ and its related analogs [colour codes: 4-Br (blue), 4-Cl (green), 4-F (yellow), 4-OH (orange), 4-Me (maroon), 4-OMe (pink) and 4-NMe 2 (purple)].

$59.3(1)^{\circ}$, respectively. Rings $\mathrm{B}$ and $\mathrm{C}$ are oriented at an angle of $40.2(1)^{\circ}$. The geometric parameters of the $\mathrm{O} 2=\mathrm{C} 9-\mathrm{C} 2=\mathrm{C} 3-\mathrm{O} 1$ and $\mathrm{O} 4=\mathrm{C} 17-\mathrm{C} 10=\mathrm{C} 11-\mathrm{O} 3$ moieties indicate the $\pi$-conjugation. One of the characteristic features in this class of compounds is the orientation of the carbon atom bearing dimethyl group. In most of the cases, this carbon atom is directed towards the phenyl ring (A) as observed in the compound $\mathbf{I}$. In the case of 4-F compound (refcode: QIKBOB) and unsubstituted compound (refcode: TAHDAV), there are two molecules in the asymmetric unit in each case. In one of the molecules, the carbon atom bonded to dimethyl group is bent away from the phenyl ring.

A CSD search was conducted using the unsubstituted compound (4-H) as a template. The search revealed that there are 18 hits found in the database of which one of the structures (3-OH derivative) contains a water molecule (refcode: GURFOO). These hits are reduced to 10 if considered only 4-substituted derivatives and some of these derivatives have multiple entries in the database. The molecular conformation is described by three different torsion angles $\left(\tau_{1}, \tau_{2}\right.$, and $\left.\tau_{3}\right)$ (Scheme 1). These three angles in the compound $\mathbf{I}$ are compared to other 4-substituted and unsubstituted structures (Table S1, Supporting Information). The structural superimposition is carried out to identify the orientation of the phenyl ring upon different substitutions. It is found that all three torsion angles are nearly the same in the structures of $4-\mathrm{NMe}_{2}, 4-\mathrm{OH}, 4-\mathrm{Me}, 4-\mathrm{OMe}$ and in the compound $\mathbf{I}(4-\mathrm{Br})$. The conjugated $\mathrm{O} 2=\mathrm{C} 9-\mathrm{C} 2=\mathrm{C} 3-\mathrm{O} 1$ and $\mathrm{O} 4=\mathrm{C} 17-\mathrm{C} 10=\mathrm{C} 11-\mathrm{O} 3$ moieties are used for
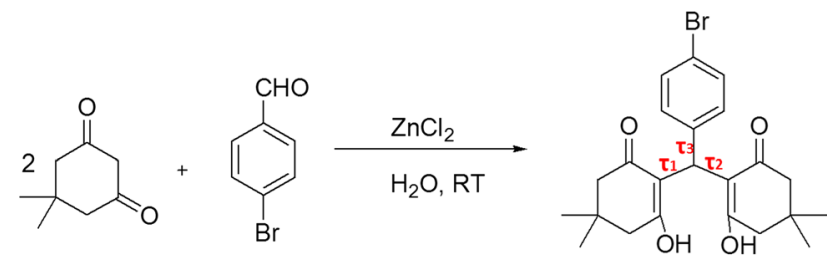

(I)

Scheme 1. Synthetic route for title compound I. Three important torsion angles $\left(\tau_{1}, \tau_{2}\right.$ and $\left.\tau_{3}\right)$ are indicated.

structural superimposition (Figure 1(b)). The maximum root mean squared deviation (rmsd: $0.19 \AA$ ) is observed for the compound I and 4-Cl (refcode: PUFTEP01) pair. Since, the sign of all three torsion angles are opposite in the case of 4-F (molecule A), 4-OET, 4-NO $\mathrm{N}_{2}$ and unsubstituted compound when compared to the compound $\mathbf{I}$, the structural superimposition has not been carried out for these molecules.

\subsection{Hirshfeld surface (HS) analysis}

Various intermolecular interactions observed in the crystal structures of $\mathbf{I}$ and its related analogs were quantified using Hirshfeld surface analysis. The HS of the compound $\mathbf{I}$ is mapped with $d_{\text {norm }}$ and the 2D fingerprint plots are shown in Figure 2. The analysis suggests that the intermolecular $\mathrm{H}$... H contacts are contributing more $(53.1 \%)$ to the crystal packing when compared to the other contacts present in the crystal structure of $\mathbf{I}$. 

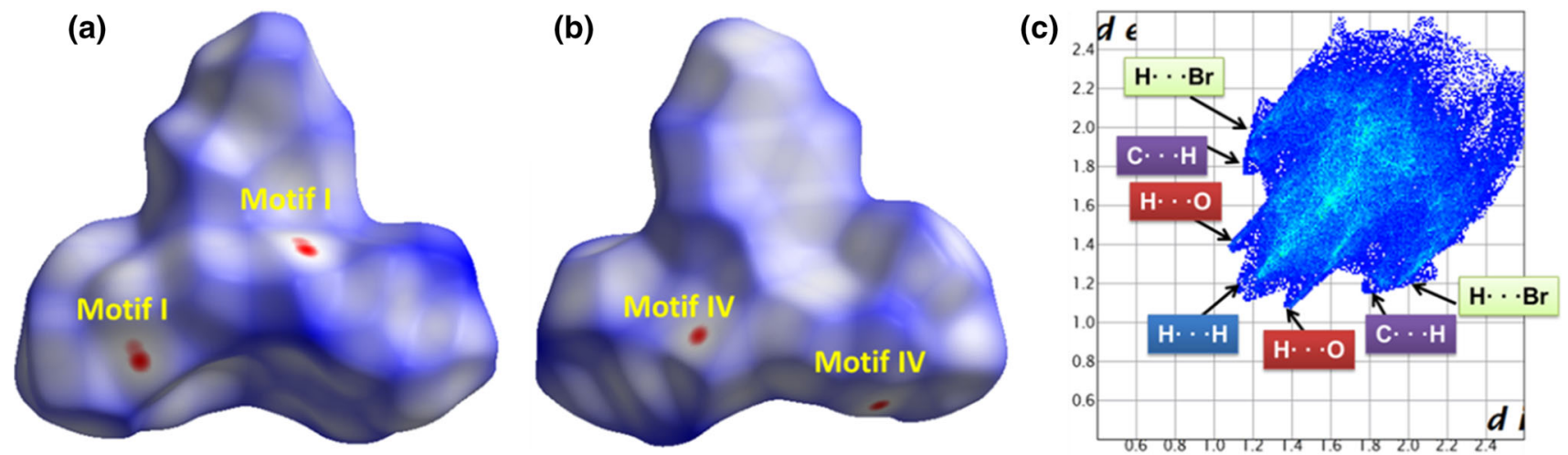

Figure 2. Hirshfeld surface (HS) analysis and two-dimensional fingerprints for the compound I (a) two different views of HS mapped with $d_{\text {norm }}$ distances (b) two-dimensional fingerprints plot showing different intermolecular contacts.

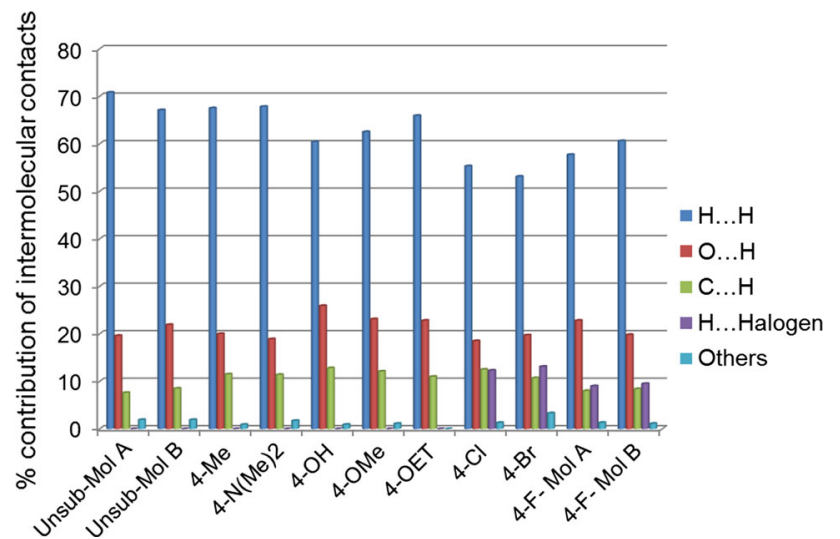

Figure 3. The relative contributions of various intermolecular interactions in the compound $\mathbf{I}$ and its related analogs.

The relative contributions of other intermolecular contacts such as $\mathrm{O} \cdots \mathrm{H} / \mathrm{H} \cdots \mathrm{O}, \mathrm{H} \cdots \mathrm{Br} / \mathrm{H} \cdots \mathrm{Br}$ and $\mathrm{C} \cdots \mathrm{H} / \mathrm{H} \cdots \mathrm{C}$ are $19.7,13.1$ and $10.7 \%$, respectively, to the total HS area of the molecule. Figure 3 displays the percentage contribution of various intermolecular contacts observed in the compound $\mathbf{I}$ and its related analogs. It is clearly showing that the intermolecular $\mathrm{H} \cdots \mathrm{H}$ contacts are predominant in all 4-substituted structures. Notably, the intermolecular $\mathrm{H}$. . H contacts are contributing more (greater than 60\%) to the crystal packing in the unsubstituted (4-H), 4-Me, 4-N(Me) $)_{2}$, 4-OH, 4-OMe, 4-OET and one of the molecules of 4$\mathrm{F}$ structures. In the case of $\mathbf{I}(4-\mathrm{Br}), 4-\mathrm{Cl}$ and one of the molecules of 4-F structures, these contacts are less than $60 \%$. Overall, there are slight variations observed for intermolecular $\mathrm{C} \cdots \mathrm{H}$ and $\mathrm{O} \cdots \mathrm{H}$ contacts due to different substituents at the para position. Two different orientations of Hirshfeld surfaces mapped with different properties such as $d_{\mathrm{i}}, d_{\mathrm{e}}, d_{\text {norm }}$, shape index and curvedness of the compound $\mathbf{I}$ and its related analogs are given in Figure S1.

\subsection{Molecular pairs in the crystal structure}

To quantify all possible intermolecular interactions present in the compound $\mathbf{I}$, the important molecular pairs (Figure 4) were retrieved from the crystal packing and their interaction strengths were quantified using the PIXEL method. Table 2 shows all possible intermolecular interactions (motif I to VI) along with their interactions energies. The interaction energies for these motifs are compared with the energies derived from DFT method.

In the crystal structure of $\mathbf{I}$, one of the methylene (via $\mathrm{H} 12 \mathrm{~B}$ ) groups (atom $\mathrm{C} 12$ ) of ring $\mathrm{C}$ is participating in an intermolecular $\mathrm{C}-\mathrm{H} \cdots \mathrm{O}$ interaction with the carbonyl oxygen atom $(\mathrm{O} 2)$ of ring $\mathrm{B}$ (motif I). This interaction links the molecules into a chain which runs parallel to the crystallographic $c$ axis. The stabilization energy for this motif I was found to be -9.1 $\mathrm{kcal} \mathrm{mol}^{-1}$ as evaluated from the PIXEL calculation. As shown in Figure 5, it is clearly seen that the major contributor for the stabilization of motif $\mathrm{I}$ is the dispersion interactions $(66.7 \%)$. Motif II is stabilized by a $\pi \cdots \pi$ stacking interaction between 4-bromophenyl rings. The dispersion interactions contribute nearly $75 \%$ towards the stabilization of motif II. The interaction energy for this motif was calculated to be $-8.9 \mathrm{kcal} \mathrm{mol}^{-1}$. Motif III is formed by an intermolecular H12B . . H6A interaction and dispersion component contributes about $83 \%$ towards the stabilization. The interaction energy for this dimer was $-7.2 \mathrm{kcal} \mathrm{mol}^{-1}$. Motif IV is generated by an intermolecular $\mathrm{C} 16-\mathrm{H} 16 \mathrm{~A} \cdots \mathrm{O} 1$ interaction. This intermolecular interaction interconnects the molecules into a chain that runs parallel to the $c$ axis. The dimer form by motif IV score an interaction energy of $-6.0 \mathrm{kcal}$ $\mathrm{mol}^{-1}$ and this dimer is stabilized by the contribution of the dispersion $(66 \%)$ and the electrostatic interactions (34\%). Furthermore, motifs I and IV are combined to form a tetrameric supramolecular motif as shown 

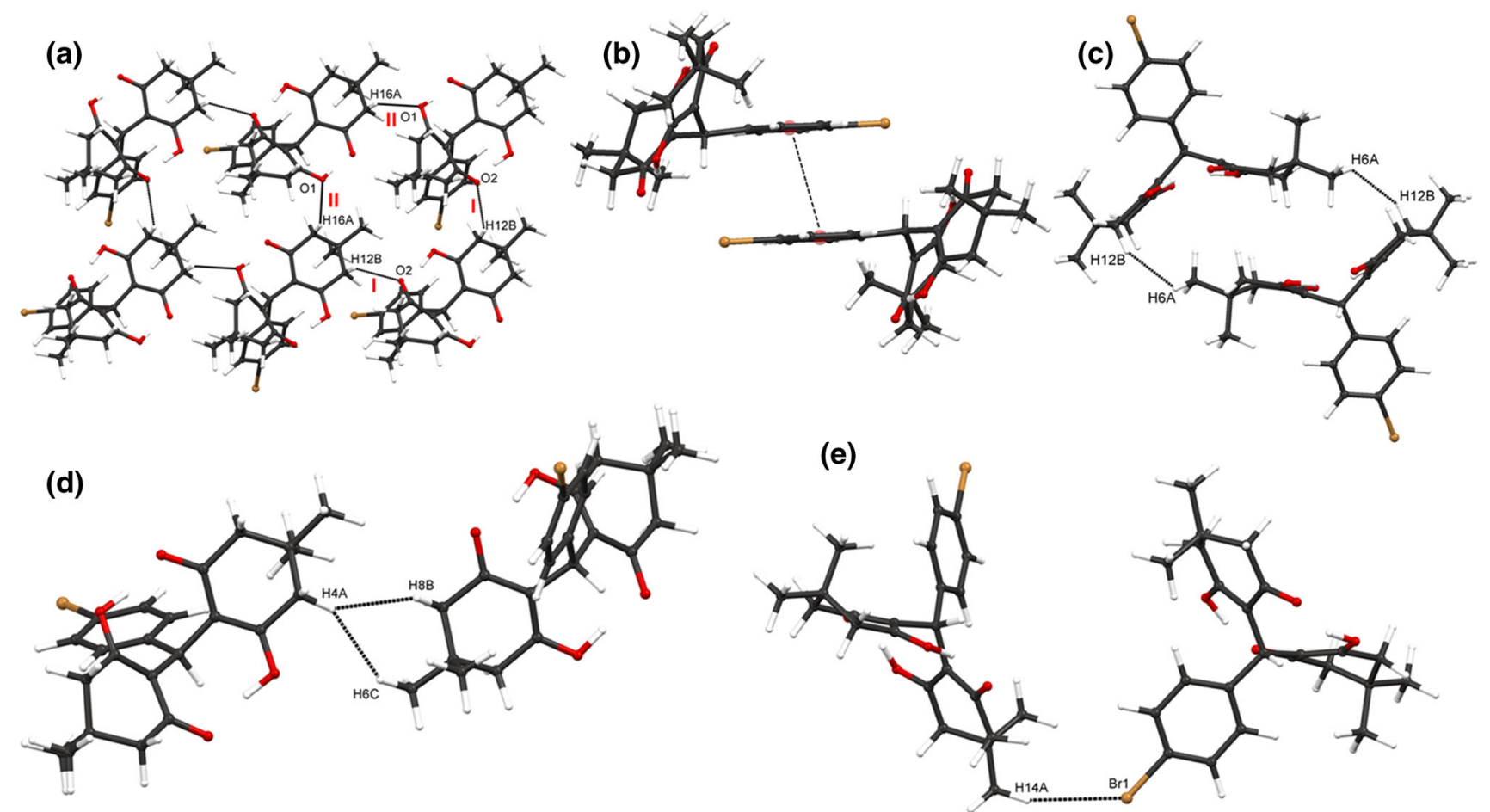

Figure 4. Selected molecular pairs extracted from the crystal structure of I. (a) Motifs I and IV (b) Motif- II (c) Motif-III (d) Motif-V and (e) Motif-VI (see Table 1 for motif details).

in Figure 4(a) and these two interactions are clearly visible on the HS (Figure 2(a-b)). Motif $\mathrm{V}$ is formed by the presence of two intermolecular $\mathrm{H} \cdots \mathrm{H}$ interactions ( $\mathrm{H} 4 \mathrm{~A} \cdots \mathrm{H} 6 \mathrm{C}$ and $\mathrm{H} 4 \mathrm{~A} \cdots \mathrm{H} 8 \mathrm{~B})$. It should be noted that the former contact is longer by $0.05 \AA$, while the latter is longer by $0.08 \AA$ when compared to the sum of the van der Waals radii of respective interacting atoms. The stabilization energy for this molecular pair was calculated to be $-3.0 \mathrm{kcal} \mathrm{mol}^{-1}$. One of the methyl groups (via $\mathrm{H} 14 \mathrm{~A}$ ) is involved in an intermolecular $\mathrm{C}-\mathrm{H} \cdots \mathrm{Br}$ interaction (motif $\mathrm{VI}$ ) with the $\mathrm{Br}$ atom of the neighbouring molecule. It is of interest to note that this interaction is slightly longer $(0.08 \AA)$ than the sum of the van der Waals radii of $\mathrm{H}$ and $\mathrm{Br}$ atoms. The interaction energy for this dimer was calculated to be $-2.7 \mathrm{kcal} \mathrm{mol}^{-1}$ and this pair is primarily stabilized by the dispersion $(\sim 70 \%)$ interactions in nature.

\subsection{Lattice energies and common packing features}

Lattice energies for the compound I and its closely related analogs were computed using the PIXEL method. The lattice energies and their components for these molecules are listed in Table 3. It is found that the overall lattice energies for these compounds are in the range of -30.7 to $-37.1 \mathrm{kcal} \mathrm{mol}^{-1}$. It is worth mentioning that the overall lattice energy is stronger for the 4-OH and weaker for 4-F and 4-Me structures.
It should be noted that the contribution of dispersion energy is predominant $(61-75 \%)$ in all structures towards the stabilization. In the case of $4-\mathrm{OH}$, the contribution of dispersion energy is reduced $(61 \%)$ and the electrostatic interaction is increased to $39 \%$ due to the $\mathrm{O} \cdots \mathrm{H}$ contacts as evident from the HS analysis. Furthermore, a substantial amount of contribution comes from $\mathrm{C} \cdots \mathrm{H} / \mathrm{H} \cdots \mathrm{C}$ contacts to the overall stabilization in the title compound $\mathbf{I}$ and its closely related analogs (Figure 3). To understand the role of $\mathrm{C} \cdots \mathrm{H}$ and $\mathrm{H} \cdots$ halogen contacts in the stabilization of the crystals, we thoroughly examined the crystal packing of the title compound and its closely related analogs using the PIXEL method. Various molecular pairs for these molecules were extracted based on their intermolecular interaction energies. The selected molecular pairs stabilized by $\mathrm{C} \cdots \mathrm{H}$ and $\mathrm{H} \cdots$ halogen contacts in 8 closely related analogs are given in Figure S2. This analysis suggests that the $\mathrm{C} \cdots \mathrm{H}$ contacts are mostly playing a supportive role in the stabilization along with intermolecular $\mathrm{C}-\mathrm{H} \cdots \mathrm{O} / \mathrm{N}$ interactions. In contrast, the molecular pair is formed by intermolecular $\mathrm{H}$... halogen interaction without any significant additional non-bonded contacts. It is to be noted that these molecular pairs usually form with low interaction energy.

Further, the common packing features exist between the compound I and its closely related analogs were analyzed as mentioned in the experimental section. As 


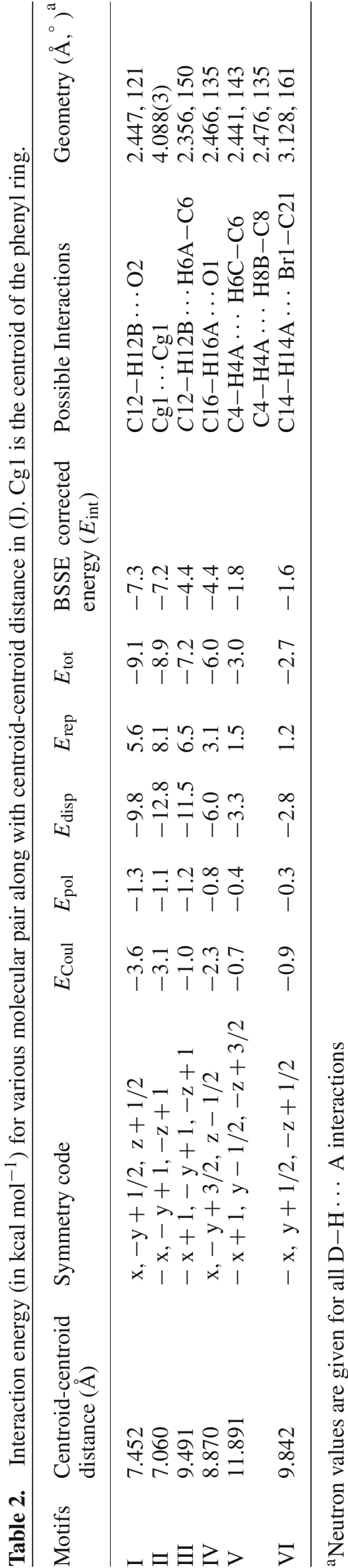

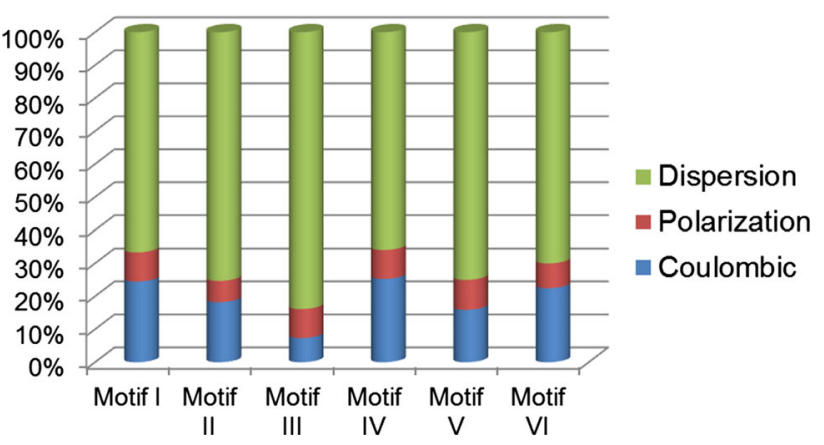

Figure 5. The relative contributions of various energy components in the different motifs of the crystal structure of $\mathbf{I}$.

shown in Figure 6, we found that the motifs II and III in the compound $\mathbf{I}$ are also observed in 4-OEt and 4-Cl structures, respectively. The former motif is stronger by $0.7 \mathrm{kcal} \mathrm{mol}^{-1}$ in the $4-\mathrm{Cl}$ structure, while the latter motif is stronger by $0.8 \mathrm{kcal} \mathrm{mol}^{-1}$ in the compound $\mathbf{I}$. This dimer match can be described as 0D similarity. Apart from this, there is no common mode of crystal packing existing between compound I and its other closely related analogs. However, the intermolecular $\mathrm{C} \cdots \mathrm{H}$ contacts help to stabilize most of the strongest dimers in this class of compound.

\subsection{DFT analysis}

To check the conformational flexibilities of $\mathbf{I}$, the monomeric structure was optimized in the gas phase as well as in the ethanol solvent using the DFT method. The optimized structures were compared with the crystal structure. The superimposed image of the DFT optimized (gas and ethanol solvent) geometry and the experimental structure indicates the bond parameters are well reproduced in the DFT calculation. The structural superimposition diagram is given in the Supplementary Information (Figure S3). The RMSD (root mean squared deviation) between X-ray and the optimized structure in the gas phase involving non-hydrogen atoms is $0.23 \AA$. The corresponding value is $0.19 \AA$ for the $\mathrm{X}$-ray and the optimized structure in ethanol solvent. A close examination of the three torsion angles $\left(\tau_{1}, \tau_{2}\right.$ and $\left.\tau_{3}\right)$ indicates that these angles are deviating by $4-20^{\circ}$ from the crystal structure geometry (Table S1). These deviations may be due to the absence of crystal packing effect in the gas and solvent phases.

\subsection{Quantitative molecular electrostatic potential}

To understand the nature of interaction that exists in the crystal packing, we analyzed the quantitative molecular electrostatic potentials (MESP) for the monomer at 
Table 3. Lattice energies (in $\mathrm{kcal} \mathrm{mol}^{-1}$ ) of the compound I and its closely related analogs (para substituted).

\begin{tabular}{lccccc}
\hline Compound & $E_{\text {Coul }}$ & $E_{\text {pol }}$ & $E_{\text {Disp }}$ & $E_{\text {Rep }}$ & $E_{\text {tot }}$ \\
\hline $4-\mathrm{H}$ & -11.0 & -4.9 & -40.1 & 24.4 & -31.6 \\
4-Br (present study) & -9.6 & -4.0 & -40.1 & 21.4 & -32.3 \\
4-Cl & -11.8 & -4.7 & -41.0 & 23.8 & -33.7 \\
$4-\mathrm{F}$ & -11.1 & -4.3 & -36.3 & 21.0 & -30.7 \\
$4-\mathrm{OH}$ & -19.6 & -8.9 & -44.3 & 35.7 & -37.1 \\
$4-\mathrm{Me}$ & -10.5 & -5.3 & -40.3 & 25.5 & -30.7 \\
4-OMe & -11.8 & -5.9 & -43.9 & 27.8 & -33.7 \\
4-OEt & -11.2 & -4.9 & -41.8 & 23.3 & -34.6 \\
4-N(Me) 2 & -11.6 & -5.6 & -46.0 & 27.5 & -35.8 \\
\hline
\end{tabular}
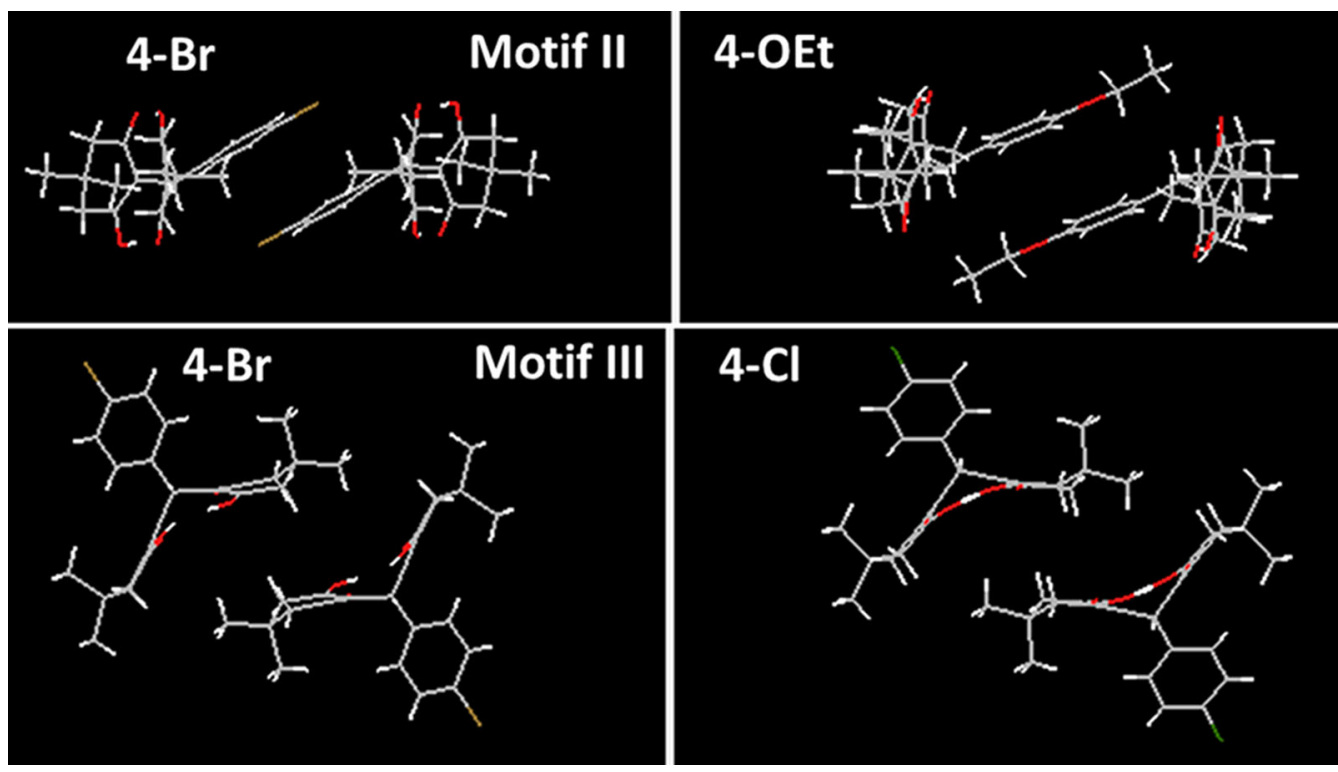

Figure 6. Common packing features observed in the compound $\mathbf{I}$ and its closely related analogs.

the 0.001 electrons/Bohr ${ }^{3}$ isodensity surface using the WFA-SAS program. ${ }^{52}$ The MESP isosurface along with various positive and negative potentials designated as $\mathrm{Vs},_{\text {max }}$ (shown in black dots) and $\mathrm{Vs}_{\text {, }}$ in (shown in blue dots) are shown in Figure 7. The most positive Vs, max value of $20.83 \mathrm{kcal} \mathrm{mol}^{-1}$ is observed on the methylene $(\mathrm{C} 16)$ hydrogen atoms neighbour to the carbonyl group and on the methylene (C4) groups neighbour to the hydroxy group. Similar values on the methylene groups (C8 and C12) attached to the carbonyl and hydroxy group show that there exists a strong bonding between them. The other positive regions are found in the dimethyl groups. The protons on the 4-bromo benzene is found to have a near positive potential of 16.69 $\mathrm{kcal} \mathrm{mol}^{-1}$ and the least value is found on the hydrogen atom attached to the tertiary carbon which links rings $\mathrm{A}, \mathrm{B}$ and $\mathrm{C}$. The most negative potential $\mathrm{Vs}_{\mathrm{s}_{\text {min }}}$ is observed on the carbonyl oxygen with a value of -31.55 $\mathrm{kcal} \mathrm{mol}^{-1}$, while the hydroxy group has $-24.85 \mathrm{kcal}$ $\mathrm{mol}^{-1}$. In addition, the negative potentials are found on the bromine atom and on the benzene ring with values $-16.39 \mathrm{kcal} \mathrm{mol}^{-1}$ and $-13.95 \mathrm{kcal} \mathrm{mol}^{-1}$, respectively. During crystal formation, the most positive regions can interact with the most negative regions of the neighboring molecule thereby leading to the directional structure formation. Thus, methylene hydrogens have a tendency to form intermolecular interactions with either carbonyl group, a hydroxyl group or with bromine atom which is evident from the PIXEL energy calculation.

\subsection{QTAIM analysis}

We performed QTAIM analysis for molecular pairs (motif I-VI; see Table 1) to confirm the existence of various intermolecular interactions and to calculate their 

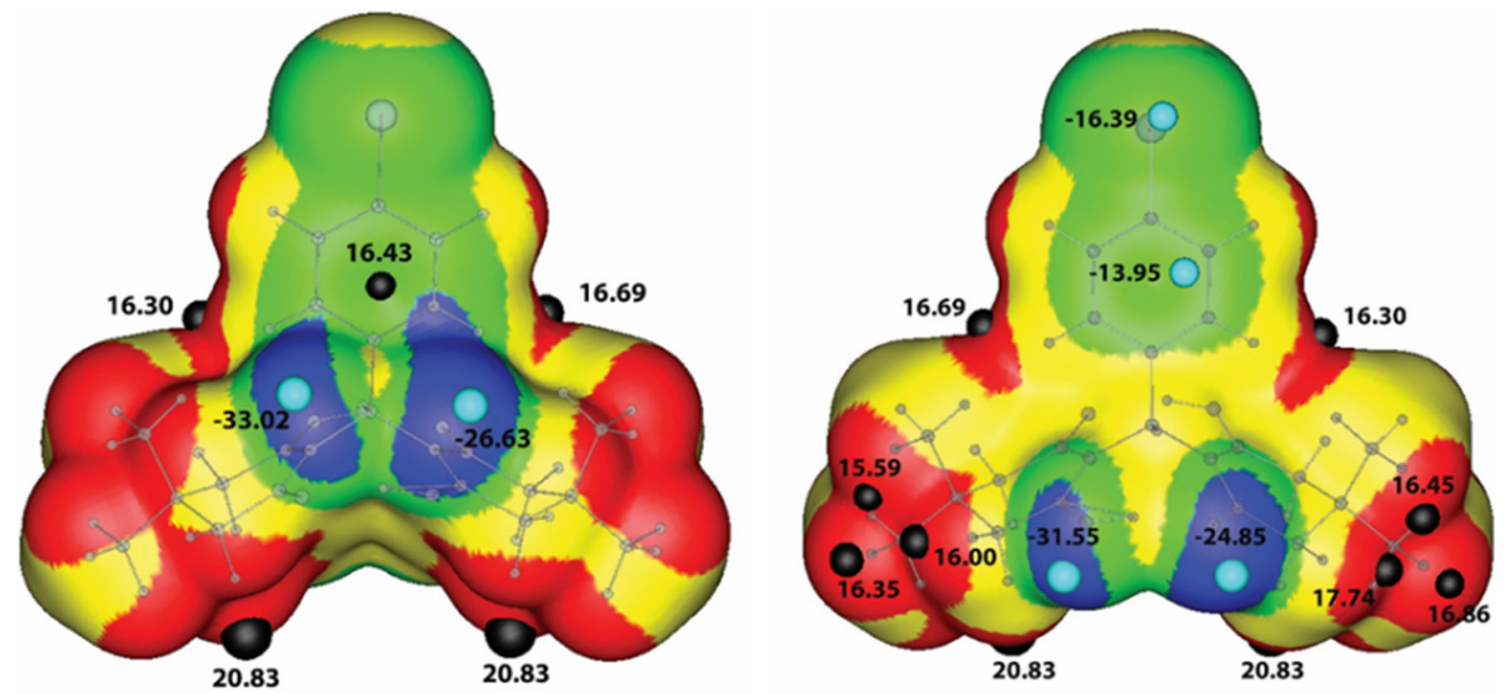

Figure 7. Molecular electrostatic potentials of the title compound displayed in two different orientations. The most positive and negative potentials are indicated.

interaction energies as proposed earlier. ${ }^{53,54}$ The molecular graphs for molecular pairs I-VI are displayed and given in the supporting information (Figure S4). The topological parameters such as the electron density $\left(\rho_{\mathrm{BCP})}\right.$, the Laplacian of electron density $\left(\nabla^{2} \rho\right)$, local potential energy density $(\mathrm{V}(\mathrm{r}))$ and kinetic energy density $(\mathrm{G}(\mathrm{r}))$ for various intermolecular interactions at the bond critical points (BCPs) are presented in Table 4 . The interaction values are computed from the local potential energy density using $E_{\mathrm{HB}}=-0.5 \mathrm{~V}(\mathrm{r})$. The $\rho_{\mathrm{BCP}}$ for the intramolecular $\mathrm{O}-\mathrm{H} \cdots$ O hydrogen bonding interactions in the optimized monomer and dimers at their crystal structure geometry lies in the range of 0.045-0.059 a.u. The $E_{\mathrm{HB}}$ was found to be in the range of $12.40-17.04 \mathrm{kcal} \mathrm{mol}^{-1}$ for these interactions. It is of interest to note that one of the intramolecular $\mathrm{O}-\mathrm{H} \cdots \mathrm{O}$ hydrogen bond is stronger by $4 \mathrm{kcal}$ $\mathrm{mol}^{-1}$ when compared to another intramolecular hydrogen bond.

In the molecular pair I, there are four intermolecular BCP's. The $\rho_{\mathrm{BCP}}$ for these interactions lie in the range of 0.004-0.010 a.u. The intermolecular H12B ‥ O2 interaction has the maximum $\rho_{\mathrm{BCP}}$ and the $E_{\mathrm{HB}}$ for this interaction was $2.34 \mathrm{kcal} \mathrm{mol}^{-1}$. The intermolecular $\mathrm{C} \cdots \mathrm{H}$ and $\mathrm{H} \cdots \mathrm{Br}$ contacts with $E_{\mathrm{HB}}$ values lie in the range of $0.62-0.75 \mathrm{kcal} \mathrm{mol}^{-1}$ which indicates their supportive role in the stabilization.

There are four symmetrical intermolecular BCPs observed in the molecular pair II. The intermolecular $\mathrm{C} 21 \cdots \mathrm{C} 22$ contact indicates the existence of $\pi \cdots \pi$ stacking. Further, the existence of $\mathrm{H} \cdots \mathrm{Br}$ and $\mathrm{Br} \cdots \mathrm{O}$ contacts provide additional stability to this molecular pair. It is of interest to note that the strength of these contacts is slightly higher than the C..C contact. The molecular pair III is formed by three symmetrical intermolecular $\mathrm{H} \cdots \mathrm{H}$ and $\mathrm{C} \cdots \mathrm{H}$ types of contacts. The intermolecular $\mathrm{H} \cdots \mathrm{H}$ contact is slightly stronger when compared to $\mathrm{C} \cdots \mathrm{H}$ contacts. In the molecular pair IV, there are four intermolecular BCPs observed. This pair is primarily stabilized by intermolecular $\mathrm{C}-\mathrm{H} \cdots \mathrm{O}$ interactions with $E_{\mathrm{HB}}$ values in the range of $2.26-0.59 \mathrm{kcal} \mathrm{mol}^{-1}$. Both hydroxy and carbonyl oxygens act as acceptors in this molecular pair. Molecular pair $\mathrm{V}$ is stabilized by intermolecular $\mathrm{H} \cdots \mathrm{H}$ type of contact and the molecular pair $\mathrm{VI}$ is formed by intermolecular $\mathrm{H} \cdots \mathrm{Br}$ type of interaction. The $E_{\mathrm{HB}}$ values for these interactions were found to be in the range of $0.61-0.53 \mathrm{kcal} \mathrm{mol}^{-1}$. Overall, the QTAIM analysis suggests that the intermolecular $\mathrm{H} \ldots \mathrm{C}$ and $\mathrm{H} \cdots \mathrm{Br}$ contacts play an important role towards the stabilization of the various molecular pairs in the crystal structure of the title compound.

\subsection{Spectral analysis}

The IR and Raman spectra recorded for the compound I to understand the nature of interactions exist in the solid state. We also computed the IR and Raman spectra for the monomer and the four most stable dimers (I-IV). A comparative IR and Raman spectra derived from experimental and the DFT method for monomer and four dimers are given in Figures S5-S6. It is clearly concluded that the computed spectra resemble the experimental spectra. In the experimental IR spectrum, we observed three intense absorption bands at 1373, 1592 and 2959 (broad) $\mathrm{cm}^{-1}$. These peaks could be assigned 


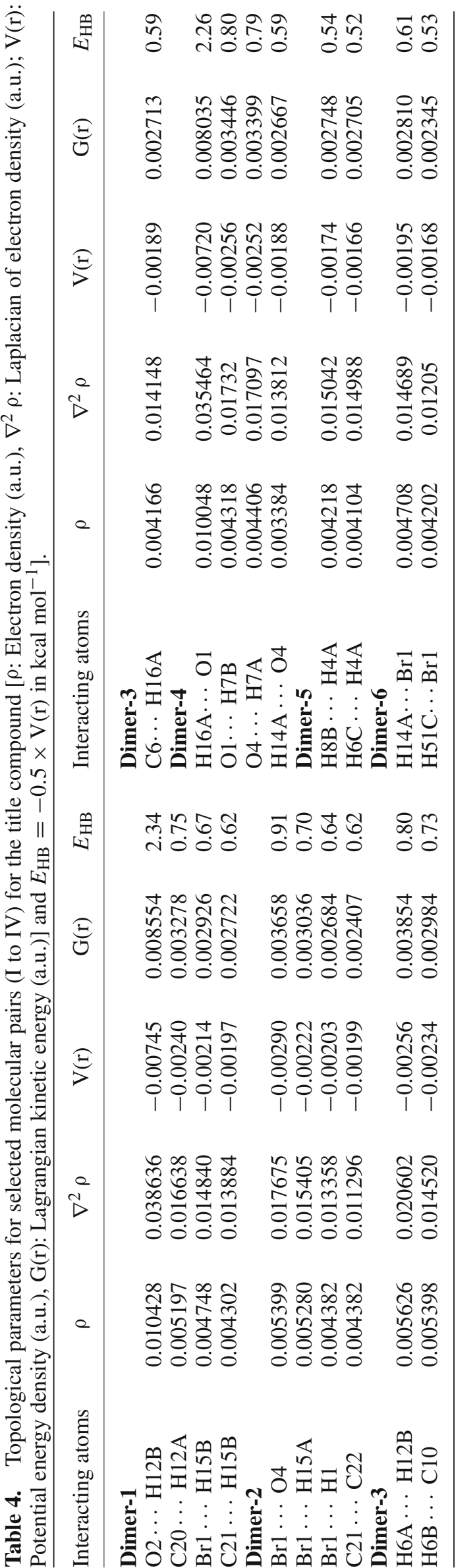

for wagging of hydroxy group, phenyl $\mathrm{C}-\mathrm{C}$ stretching and methylene $\mathrm{C}-\mathrm{H}$ and methyl $\mathrm{C}-\mathrm{H}$ stretching vibrations, respectively. It is noted that the broadness of the latter peak due to the overlap of methylene and methyl $\mathrm{C}-\mathrm{H}$ vibrations. In the crystal structure, dimer$\mathrm{I}$ is stabilized by an intermolecular $\mathrm{C} 12-\mathrm{H} 12 \mathrm{~B} \cdots \mathrm{O} 2$ interaction. The C12-H12B stretching vibration is calculated at $3067 \mathrm{~cm}^{-1}$ which is comparable with the experimental IR peak at $2962 \mathrm{~cm}^{-1}$. This blue shift is the consequence of the formation of intermolecular interaction. In dimer-II, the phenyl $\mathrm{C}-\mathrm{C}$ stretching (red shift) is calculated at 1563 and $1566 \mathrm{~cm}^{-1}$ which is responsible for a weak $\pi \cdots \pi$ interaction. Similarly, dimer-III is formed by an intermolecular C12-H12B ... H6A interaction (between methylene hydrogen and methyl hydrogen). The stretching vibration for this interaction is calculated at $3069 \mathrm{~cm}^{-1}$. The $\mathrm{C}=\mathrm{O}$ stretching vibration is calculated in the range of $1700-1728 \mathrm{~cm}^{-1}$ in the monomer and in four dimers. In dimer-IV, due to a slight conformational change on ring $\mathrm{C}$, intermolecular interactions $\mathrm{C} 12-\mathrm{H} 12 \mathrm{~B} \ldots \mathrm{O} 1$ is formed which is not observed in the crystal structure. The stretching vibration of C12-H12B bond is calculated at $3071 \mathrm{~cm}^{-1}$. In the experimental Raman spectrum, absorption peaks at $3278,3154,3090,1696$ and $1652 \mathrm{~cm}^{-1}$ observed. The peaks could be assigned for phenyl $\mathrm{C}-\mathrm{H}$ stretching, methylene $\mathrm{C}-\mathrm{H}$, methyl $\mathrm{C}-\mathrm{H}$, phenyl $\mathrm{C}-\mathrm{C}$ stretching and wagging of $\mathrm{O}-\mathrm{H}$ vibrations, respectively.

As mentioned earlier, the UV-visible spectrum for the compound I was computed in ethanol solvent using the TD-DFT method with polarizable conductor calculation model (CPCM) with Klamt sphere radii. Figure 8 shows the overlap diagram of the experimental and the simulated spectra and in Table 5 we present the excitation energies, oscillator strengths $(f)$ and the electron configurations. The experimental spectrum shows an intensive absorption peak at $260 \mathrm{~nm}$ and the corresponding peak was observed at $239 \mathrm{~nm}$ in the TD-DFT calculation. The frontier molecular orbitals involved in the transition are shown in Figure 8. The peak calculated at $263 \mathrm{~nm}$ with an oscillatory strength of 0.055 is primarily constituted by the HOMO $\rightarrow$ LUMO transition. The excitation at $239 \mathrm{~nm}$ with an oscillatory strength of 0.538 corresponds to $\mathrm{HOMO} \rightarrow \mathrm{LUMO}+1$ transition which contribute about $85 \%$. These two excitations indicate that the charge transfer HOMO $\rightarrow$ LUMO and $\mathrm{HOMO} \rightarrow \mathrm{LUMO}+1$ orbitals. The band gap energy for the former transition is $7.32 \mathrm{eV}$, while the latter transition is found to be $7.58 \mathrm{eV}$. It is of interest to note that the HOMO orbitals are localized over the bromobenzene ring and $\pi$-conjugated moieties as mentioned earlier, while the LUMO orbital is located on the conjugated moieties of the cyclohexene rings. 

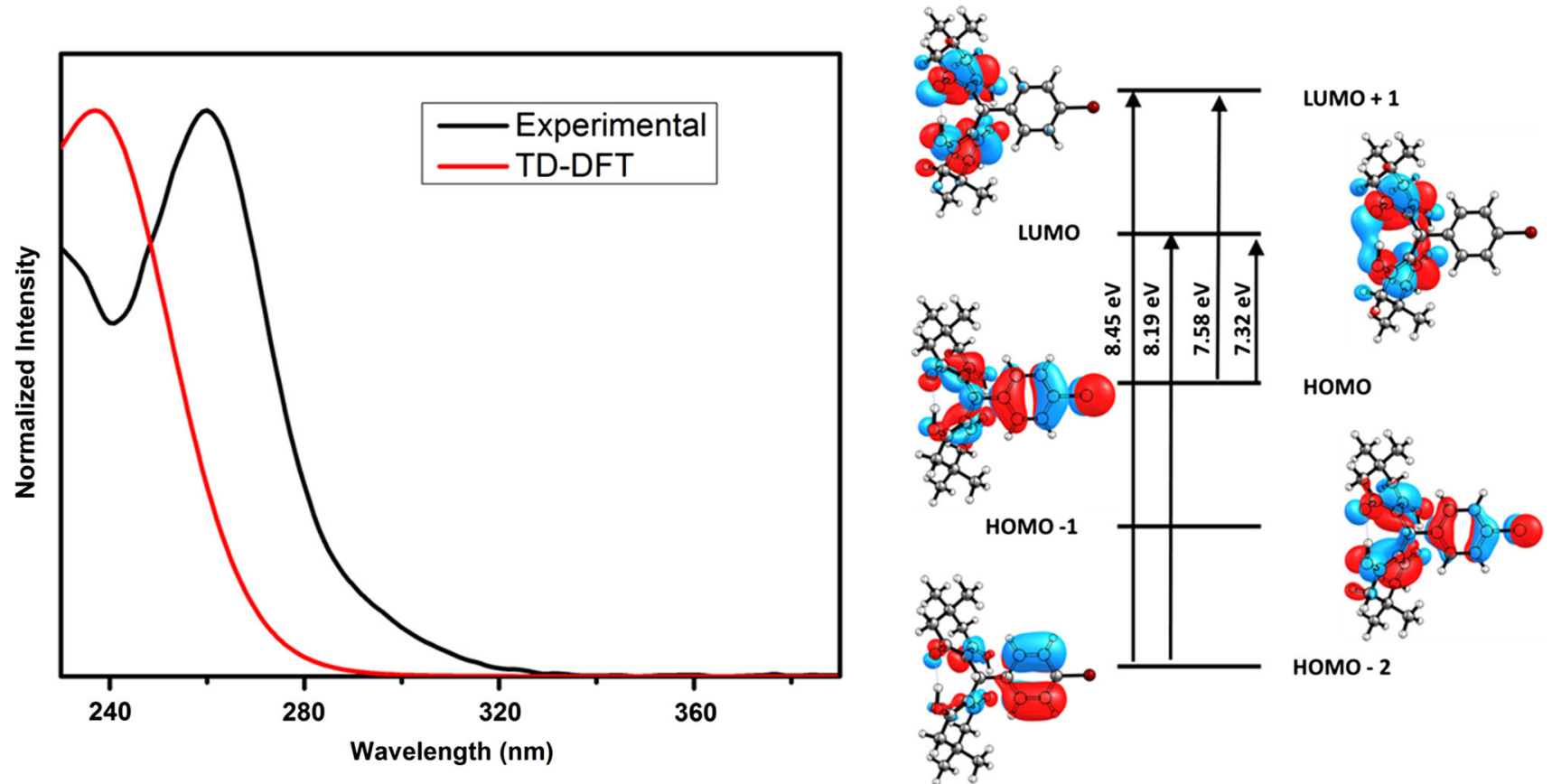

Figure 8. The observed and calculated UV-Vis spectra (left) and HOMO-LUMO orbitals (right).

Table 5. Excitation wavelengths (in nm), oscillator strengths, configurations, and experimental data for the compound $\mathbf{I}$ in ethanol solvent.

\begin{tabular}{llll}
\hline Excitation Energy & $f$ & Main configuration & Expt. \\
\hline 263 & 0.0550 & $\begin{array}{l}\text { HOMO } \rightarrow \text { LUMO (84\%) } \\
\text { HOMO - 2 } \rightarrow \text { LUMO + 1 (7\%) }\end{array}$ & 260 \\
239 & 0.5380 & HOMO $\rightarrow$ LUMO+1 (85\%) & \\
& & HOMO - 2 $\rightarrow$ LUMO (9\%) & \\
\hline
\end{tabular}

\section{Conclusions}

The compound I was synthesized and characterized by various experimental and theoretical methods. The overall conformation of the compound I was compared with its closely related analogs. The crystal structure of the compound $\mathbf{I}$ revealed that the compound exhibited two strong intramolecular $\mathrm{O}-\mathrm{H} \cdots \mathrm{O}$ hydrogen bonding interactions. Intermolecular $\mathrm{C}-\mathrm{H} \cdots \mathrm{O}, \pi \cdots \pi$ and $\mathrm{H} \cdots \mathrm{H}$ contacts were responsible for the molecular self-assembly. Further, the energetics of the various intermolecular interactions was estimated using PIXEL, DFT and QTAIM analysis. The relative contributions of various non-bonded contacts present in the compound $\mathbf{I}$ and its closely related analogs were quantified using Hirshfeld surface analysis. We found that motifs II and III of the compound I were also existed in its closely related analogs, 4-OEt and 4-Cl. Apart from this, there is no common mode of crystal packing existing between compound I and its other closely related analogs. The PIXEL, HS and QTAIM calculations collectively suggest that $\mathrm{H} \cdots \mathrm{C}$ and $\mathrm{H} \cdots$ halogen contacts play an important role towards the overall stabilization in this class of compounds. Further, FT-IR and FT-Raman spectra were derived from experimental and simulated by the DFT method. The results support the existence of various intermolecular interactions in the crystal structure. The analysis of the frontier molecular orbitals provides evidences for a charge transfer process is taking place within the molecule.

\section{Supplementary Information (SI)}

The supplementary information includes the following: Table S1 contains the selected torsion angles for the title compound and its related analogs, Figure S1 shows various properties mapped with Hirshfeld surfaces, Figure S2 displays the selected molecular pairs of 8 closely related analogs of the title compound, Figure S3 shows structural superimposition, Figure S5 depicts the molecular graphs obtained from QTAIM analysis and Figures S5-S6 provide the experimental and theoretical FT-IR and FT-Raman spectra. CCDC1543019 , contains the supplementary crystallographic data 
for the title compound I. These data can be obtained freely via http://www.ccdc.cam.ac.uk/data_request/cif or by e-mailing to data_request@ccdc.cam.ac.uk or by contacting directly the Cambridge Crystallographic Data Centre (12 Union Road, Cambridge CB2 1EZ, UK. Fax: +44 1223 336033). Supplementary Information is available at www.ias. ac.in/chemsci.

\section{Acknowledgements}

ST thanks the DST-SERB (SB/YS/LS-19/2014) for research funding. Authors would like to thank Laboratorio Nacional de Supercómputo del Sureste (LNS-BUAP) and Bioinformatics Resources and Application Facility (BRAF), C-DAC Pune for computational work. Authors thank DST-PURSE Lab, Mangalore University for providing single crystal X-ray diffraction facility. SSG thanks Science and Engineering Research Board, DST-SERB (EMR/2016/000317) and Council of Scientific and Industrial Research, CSIR (80(0085)/16/EMR-II) for financial support.

\section{References}

1. Shanmugasundram P, Prabahar K J and Ramakrishnan V T 1993 A new class of laser dyes from acridinedione derivatives J. Heterocycl. Chem. 301003

2. Shanmugasundram $P$, Murugan $P$, Ramakrishnan V T, Srividya N and Ramamurthy P 1996 Synthesis of acridinedione derivatives as laser dyes Heteroatom Chem. 717

3. Mahavi G M, Ali S, Riaz N, Afza N, Malik A, Ashraf M, Iqbal L and Lateef M 2008 Mild and efficient synthesis of new tetraketones as lipoxygenase inhibitors and antioxidants J. Enzyme Inhib. Med. Chem. 2362

4. Khan K M, Mahavi G M, Khan M T H, Shaihk A J, Perveen S, Begum S and Choudhary M I 2006 Tetraketones: a new class of tyrosinase inhibitors Bioorg. Med. Chem. 14344

5. Goodell J R, Madhok A A, Hiasa H and Ferguson D M 2006 Synthesis and evaluation of acridine- and acridonebased anti-herpes agents with topoisomerase activity Bioorg. Med. Chem. 145467

6. Lambert R W, Martin J A, Merrett J H, Parkes K E B and Thomas J G 1997 PCT Int. Appl. WO 9706178 Chem. Abstr. 126 212377y

7. Jin T-S, Wang A-Q, Ma H, Zhang J-S and Li T-S 2006 The reaction of aromatic aldehydes and 5,5-dimethyl 1,3cyclohexandione under solvent free grinding conditions Indian J. Chem. 45 B 470

8. Li J-T, Li Y-W, Song Y-L and Chen G-F 2012 Improved synthesis of 2,2'-arylmethylene bis(3-hydroxy-5,5dimethyl-2-cyclohexene-1-one) derivatives catalyzed by urea under ultrasound Utrason. Sonochem. 191

9. Ganesan S S, Kothandapani J and Ganesan A 2014 Zinc chloride catalyzed collective synthesis of arylmethylene bis(3-hydroxy-2-cyclohexene-1-one) and 1,8-dioxo-octahydroxanthene/acridine derivatives Lett. Org. Chem. 11682

10. Ilangovan A, Muralidharan S, Sakthivel P, Malayappasamy S, Karuppusamy S and Kaushik M P 2013
Simple and cost effective acid catalysts for efficient synthesis of 8-aryl-1,8-dioxooctahydroxanthene Tetrahedron Lett. $\mathbf{5 4} 491$

11. Ilangovan A, Malayappasamy S, Muralidharan S and Maruthamuthu S 2011 A highly efficient green synthesis of 1,8-dioxo-octahydroxanthenes Chem. Cent. J. 581

12. Vaid R, Gupta S, Kant R and Gupta V K 2016 Domino Knoevenagel/Michael synthesis of 2,2'arylmethylene bis(3-hydroxy-5,5-dimethyl-2-cyclohexen-1-one) derivatives catalyzed by silica-diphenic acid and their single crystal X-ray analysis J. Chem. Sci. 128967

13. Sughanya V and Sureshbabu N 2012 2,2'-[3-Bromo-4hydoxy-5-methoxyphenyl)methylidene]bis(3-hydroxy5,5-dimethylcyclohex-2-en-1-one Acta Cryst. $\quad$ E68 o 2875

14. Sureshbabu N and Sughanya V 2012 2,2'-[(4-Ethoxyphenyl)methylene]bis(3-hydroxy-5,5-dimethylcyclohex-2-en-1one) Acta Cryst. E68 o2638

15. Bolte M, Degen A, Rühl S 1997 Twinned crystal structure of bis(2-hydroxy-4,4-dimethyl-6-oxo-1cyclohexenyl)phenylmethane at $150 \mathrm{~K}$ Acta Cryst. $\mathbf{C 5 3}$ 340

16. Bolte M, Degen A and Rühl S 2001 2,2'-[(3-Hydroxyphenyl)methylene]bis(3-hydroxy-5,5-dimethyl-2-cyclohexen-1-one) hydrate Acta Cryst. E57 o170

17. Bolte M, Degen A and Rühl S 2001 Packing considerations of bis-dimedone derivatives Acta Cryst. C57 446

18. Yang X-H, Zhou Y-H, Zhang M and Hu L-H 2011 3Hydroxy-2-[(4-hydroxy-3,5-dimethoxyphenyl)(2-hydroxy-4,4-dimethyl-6-oxocyclohex-1-en-1-yl)methyl]-5, 5-dimethylcyclohex-2-en-1-one Acta Cryst. E67 o492

19. Palakshi Reddy B, Vijayakumar V, Sarveswari S, Ng S W and Tiekink E R T 2010 3-Hydroxy-2-[(2-hydroxy-4,4dimethyl-6-oxocyclohex-1-en-1-yl)(3-nitrophenyl)methyl]-5,5-dimethylclclohex-2-en-1-one Acta Cryst. E66 o2806

20. Cagulada A M, Lynch D E and Hamilton D G 2009 Structure and properties of modular components for applications in topological supramolecular chemistry Cryst. Growth Des. 9825

21. Panini P and Chopra D 2012 Role of intermolecular interactions involving organic fluorine in trifluoromethylated benzanilides CrystEngComm 141972

22. Panini P and Chopra D 2013 Quantitative insights into energy contributions of intermolecular interactions in fluorine and trifluoromethyl substituted isomeric $\mathrm{N}$ phenylacetamides and $N$-methylbenzamides CrystEngComm 153711

23. Desiraju G R $2005 \mathrm{C}-\mathrm{H} \cdots \mathrm{O}$ and other weak hydrogen bonds. From crystal engineering to virtual screening Chem. Commun. 2995

24. Thomas S P, Pavan M S and Guru Row T N 2012 Charge density analysis of ferulic acid: Robustness of a trifurcated C - H . . O hydrogen bond Cryst. Growth Des. 12 6083

25. Kaur G, Panini P, Chopra D and Choudhury A R 2012 Structural investigation of weak intermolecular interactions in fluorine substituted isomeric Nbenzylideneanilines Cryst. Growth Des. 125096

26. Karanam M and Choudhury A R 2013 Study of halogenmediated weak interactions in a series of halogen substituted azobenzenes Cryst. Growth Des. 134803 
27. Nishio M 2011 The $\mathrm{CH} / \pi$ hydrogen bond in chemistry. Conformation, supramolecules, optical resolution and interactions involving carbohydrates Phys. Chem. Chem. Phys. 1313873

28. Gonthier J F, Steinmann S N, Roch L, Ruggi A, Luisier N, Severin K and Corminboeuf C $2012 \pi$-depletion as a criterion to predict $\pi$-stacking ability Chem. Commun. 48 9239

29. Bader R W F 1994 Atoms in Molecules: A Quantum Theory, Oxford University Press, USA

30. Bader R W F 1991 A quantum theory of molecular structure and its applications Chem. Rev. 91893

31. Altomare A, Cascarano G, Giacovazzo C, Guagliardi A, Burla M C, Polidori G and Camalli M 1994 SIR92-a program for automatic solution of crystal structures by direct methods J. Appl. Cryst. 27435

32. Sheldrick G M 2015 Crystal structure refinement with SHELXL Acta Cryst. C71 3

33. Macrae C F, Edgington P R, McCabe P, Pidcock E, Shields G P, Taylor R, Towler M, van de Streek J 2006 Mercury: visualization and analysis of crystal structures J. Appl. Cryst. 39453

34. Wolff S K, Grimwood G J, McKinnon J J, Turner M J, Jayatilaka D and Spackman M A 2012 CrystalExplorer, Version 3.0, University of Western, Australia

35. Gavezzotti A 2011 Efficient computer modeling of organic materials. The atom-atom, Coulomb-LondonPauli (AA-CLP) model for intermolecular electrostaticpolarization, dispersion and repulsion energies New J. Chem. 351360

36. Gavezzotti A 2003 Calculation of intermolecular interaction energies by direct numerical integration over electron densities. 2. An improved polarization model and the evaluation of dispersion and repulsion energies J. Phys. Chem. B107 2344

37. Gavezzotti A 2002 Calculation of intermolecular interaction energies by direct numerical integration over electron densities. I. Electrostatic and polarization energies in molecular crystals J. Phys. Chem. B106 4145

38. Venkatesan P, Thamotharan S, Ganesh Kumar R and Ilangovan A 2015 Invariant and variable intermolecular interactions in functionalized malonic acid half-esters: X-ray, Hirshfeld surface and PIXEL energy analyses CrystEngComm 17904

39. Venkatesan P, Thamotharan S, Ilangovan A, Liang $\mathrm{H}$ and Sundius T 2016 Crystal structure, Hirshfeld surfaces and DFT computation of NLO active (2E)-2-(ethoxycarbonyl)-3-[(1-methoxy-1-oxo3-phenylpropan-2-yl)amino]prop-2-enoic acid Spectrochim. Acta A153 625

40. Venkatesan P, Rajakannan V, Venkataramanan N S, Ilangovan A, Sundius T and Thamotharan S 2016 Structural investigation of (2E)-2-(ethoxycarbonyl)-3-[(4methoxyphenyl)amino]prop-2-enoic acid: X-ray crystal structure, spectroscopy and DFT J. Mol. Struct. 1119 259

41. Udayakumar M, Jagatheeswaran K, Ganesan S S, Venkataramanan N S, Madan Kumar S and Byrappa K 2017 Investigation of 9-(2-hydroxy-4,4-dimethyk-6oxocyclohex-1-en-1yl)-3,3-dimethyl-2,3,4,9-tetrahydro -1H-xanthen-1-one: Crystal structure, AIM and NBO analysis J. Mol. Struct. 1133510
42. Frisch M J, Trucks G W, Schlegel H B, Scuseria G E, Robb M A, Cheeseman J R, Montgomery Jr. J A, Vreven T, Kudin K N, Burant J C, Millam J M, Iyengar S S, Tomasi J, Barone V, Mennucci B, Cossi M, Scalmani G, Rega N, Petersson G A, Nakatsuji H, Hada M, Ehara M, Toyota K, Fukuda R, Hasegawa J, Ishida M, Nakajima T, Honda Y, Kitao O, Nakai H, Klene M, Li X, Knox J E, Hratchian H P, Cross J B, Adamo C, Jaramillo J, Gompert R, Stratmann R E, Yazyev O, Austin A J, Cammi R, Pomelli C, Ochterski J W, Ayala P Y, Morokuma K, Voth G A, Salvador P, Dannenberg J J, Zakrzewski V G, Dapprich S, Daniels A D, Strain M C, Farkas O, Malick D K, Rabuck A D, Raghavachari K, Foresman J B, Ortiz J V, Cui Q, Baboul A G, Clifford S, Cioslowski J, Stefanov B B, Liu G, Liashenko A, Piskorz P, Komaromi I, Martin R L, Fox D J, Keith T, Al-Laham M A, Peng C Y, Nanayakkara A, Challacombe M, Gill P M W, Johnson B, Chen W, Wong M W, Gonzalez C and Pople J A 2009 Gaussian 09 Revision B 05 Pittsburgh PA: Program, Gaussian Inc., Wallingford

43. Gelbrich T and Hursthouse M B 2005 A versatile procedure for the identification, description and quantification of structural similarity in molecular crystals CrystEngComm 7324

44. Gelbrich T and Hursthouse M B 2006 Systematic investigation of the relationships between 25 crystal structures containing the carbamazepine molecule or a close analogue: a case study of the XPac method CrystEngComm 8448

45. Gelbrich T, Threlfall T L and Hursthouse M B 2012 XPac dissimilarity parameters as quantitative descriptors of isostructurality: the case of fourteen 4,5 '-substituted benzenesulfonamido-2-pyridines obtained by substituent interchange involving $\mathrm{CF}_{3} / \mathrm{I} / \mathrm{Br} / \mathrm{Cl} / \mathrm{F} / \mathrm{Me} / \mathrm{H}$ CrystEngComm 145454

46. Zhao Y, Schultz N E and Truhlar D G 2006 Design of density functionals by combining the method of constraint satisfaction with parametrization for thermochemistry, thermochemical kinetics and noncovalent interactions $J$. Chem. Theory Comput. 2364

47. Venkataramanan N S 2016 Cooperativity of intermolecular hydrogen bonds in microsolvated DMSO and DMF clusters: a DFT, AIM and NCI analysis J. Mol. Model. 22151

48. Boys S F and Bernardi F 1970 The calculation of small molecular interactions by the differences of separate total energies. Some procedures with reduced errors $\mathrm{Mol}$. Phys. 19553

49. Scalmani G, Frish M J, Mennucci B, Tomsai J, Cammi $\mathrm{R}$ and Barone V 2006 Geometries and properties of excited states in the gas phase and in solution: Theory and application of a time-dependent density functional theory polarizable continuum model J. Chem. Phys. 124 94107

50. Todd A K 2017 AIMALL version 16.05.18, TK Gristmill Software, Overland Park KS, USA

51. Cremer D and Pople J A 1975 General definition of ring puckering coordinates J. Am. Chem. Soc. 97 1354

52. Bulat F A, Toro-Labbé A, Brinck T, Murray J S and Politzer P 2010 Quantitative analysis of molecular surfaces: areas, volumes, electrostatic potentials and 
average local ionization energies J. Mol. Model. 16 1679

53. Espinosa E, Molins E and Lecomte C 1998 Hydrogen bond strengths revealed by topological analyses of experimentally observed electron densities Chem. Phys. Lett. 285170
54. Mata I, Alkorta I, Espinosa E and Molins E 2011 Relationships between interaction energy, intermolecular distance and electron density properties in hydrogen bonded complexes under external electric fields Chem. Phys. Lett. $\mathbf{5 0 7}$ 185 